\title{
Canakinumab relieves symptoms of acute flares and improves health-related quality of life in patients with difficult-to-treat Gouty Arthritis by suppressing inflammation: results of a randomized, dose-ranging study
}

Naomi Schlesinger ${ }^{1 *}$, Marc De Meulemeester ${ }^{2}$, Andrey Pikhlak $^{3}$, A Eftal Yücel ${ }^{4}$, Dominik Richard ${ }^{5}$, Valda Murphy $^{5}$, Udayasankar Arulmani ${ }^{5}$, Peter Sallstig ${ }^{5}$ and Alexander So ${ }^{6}$

\begin{abstract}
Introduction: We report the impact of canakinumab, a fully human anti-interleukin-1 $\beta$ monoclonal antibody, on inflammation and health-related quality of life (HRQoL) in patients with difficult-to-treat Gouty Arthritis.

Methods: In this eight-week, single-blind, double-dummy, dose-ranging study, patients with acute Gouty Arthritis flares who were unresponsive or intolerant to - or had contraindications for - non-steroidal anti-inflammatory drugs and/or colchicine were randomized to receive a single subcutaneous dose of canakinumab (10, 25, 50, 90, or 150 $\mathrm{mg})(N=143)$ or an intramuscular dose of triamcinolone acetonide $40 \mathrm{mg}(N=57)$. Patients assessed pain using a Likert scale, physicians assessed clinical signs of joint inflammation, and HRQoL was measured using the 36-item Short-Form Health Survey (SF-36) (acute version).

Results: At baseline, $98 \%$ of patients were suffering from moderate-to-extreme pain. The percentage of patients with no or mild pain was numerically greater in most canakinumab groups compared with triamcinolone acetonide from 24 to 72 hours post-dose; the difference was statistically significant for canakinumab $150 \mathrm{mg}$ at these time points $(P<0.05)$. Treatment with canakinumab $150 \mathrm{mg}$ was associated with statistically significant lower Likert scores for tenderness (odds ratio $(\mathrm{OR}), 3.2 ; 95 \%$ confidence interval $(\mathrm{Cl}), 1.27$ to $7.89 ; P=0.014$ ) and swelling $(\mathrm{OR}, 2.7 ; 95 \% \mathrm{Cl}, 1.09$ to $6.50, P=0.032)$ at 72 hours compared with triamcinolone acetonide. Median C-reactive protein and serum amyloid A levels were normalized by seven days post-dose in most canakinumab groups, but remained elevated in the triamcinolone acetonide group. Improvements in physical health were observed at seven days post-dose in all treatment groups; increases in scores were highest for canakinumab $150 \mathrm{mg}$. In this group, the mean SF-36 physical component summary score increased by 12.0 points from baseline to 48.3 at seven days post-dose. SF-36 scores for physical functioning and bodily pain for the canakinumab $150 \mathrm{mg}$ group approached those for the US general population by seven days post-dose and reached norm values by eight weeks post-dose.

Conclusions: Canakinumab $150 \mathrm{mg}$ provided significantly greater and more rapid reduction in pain and signs and symptoms of inflammation compared with triamcinolone acetonide $40 \mathrm{mg}$. Improvements in HRQoL were seen in both treatment groups with a faster onset with canakinumab $150 \mathrm{mg}$ compared with triamcinolone acetonide $40 \mathrm{mg}$.

Trial registration: clinicaltrials.gov: NCT00798369.
\end{abstract}

\footnotetext{
* Correspondence: schlesna@umdnj.edu

'Division of Rheumatology, Department of Medicine, Robert Wood Johnson

Medical School, 125 Patterson Street, New Brunswick, NJ 089010, USA

Full list of author information is available at the end of the article
} 


\section{Introduction}

Gouty arthritis is a common inflammatory arthritis caused by the deposition of monosodium urate (MSU) crystals in joints $[1,2]$. The deposition and dissolution of the MSU crystals themselves depends on serum urate levels. MSU crystals induce secretion of interleukin-1 $\beta$ (IL-1 $\beta$ ), a proinflammatory cytokine that mediates the inflammation that is characteristic of Gouty Arthritis flares and that may remain present between flares $[3,4]$. There is evidence to suggest that IL-1 $\beta$ may also contribute to joint destruction in Gouty Arthritis [5-7]. Current treatments to control the pain and inflammation associated with acute flares include non-steroidal anti-inflammatory drugs (NSAIDs), colchicine, and corticosteroids $[8,9]$. However, these treatments are not always effective [10]. In addition, many patients with Gouty Arthritis have underlying comorbidities such as cardiovascular disease, diabetes mellitus, hypertension, liver and renal disease, or gastrointestinal problems, which restrict treatment options or necessitate modifications in the management of Gouty Arthritis [8,11-14]. Furthermore, patients with comorbidities often experience frequent flares. Therefore, there is a need for new therapies to provide effective pain relief in these patients with difficult-to-treat Gouty Arthritis.

Without adequate treatment, Gouty Arthritis can progress into a chronic, deforming, and physically disabling disease through the development of disfiguring tophi, joint destruction, and persistent pain $[15,16]$. Pain and impaired physical functioning can have a major impact on a patient's health-related quality of life (HRQoL). Results from several observational studies in patients experiencing frequent Gouty Arthritis flares reported HRQoL scores considerably lower than those for men of a similar age in the US general population [17-21]. In addition, patients who experienced more Gouty Arthritis flares and had a greater number of joints involved had a particularly poor HRQoL $[18,19]$. However, there are no published data regarding the impact of acute flares on HRQoL. The unpredictability of Gouty Arthritis flares further accentuates the impact of this disease on patients. If inappropriately managed, Gouty Arthritis represents a considerable economic burden through lost productivity and the cost of treatment, especially in patients with frequent flares [22,23].

Results of a recent multicenter phase 2 study have shown that canakinumab, a fully human anti-IL-1 $\beta$ monoclonal antibody, can produce rapid reductions in pain in patients with Gouty Arthritis who are unresponsive or intolerant to, or contraindicated for NSAIDs and/or colchicine, and can significantly reduce the risk of recurrent flares [24]. The canakinumab $150 \mathrm{mg}$ dose was found to be superior to triamcinolone acetonide $40 \mathrm{mg}$ on all efficacy measures reported [24]. Here we report further results from this study which confirm the superiority of the $150 \mathrm{mg}$ dose over triamcinolone acetonide including effects on pain assessed using the Likert scale, clinical signs and markers of inflammation (C-reactive protein (CRP) and serum amyloid A protein (SAA)), as well as improvements in HRQoL measures (36-item Short-Form Health Survey (SF-36) and Health Assessment Questionnaire (HAQ)).

\section{Materials and methods Study design}

This was an adaptive single-dose, single-blind, activecontrolled study. The study was approved by each local independent ethics committee. It was performed in concordance with the ICH Harmonised Tripartite Guidelines for Good Clinical Practice and the ethical principles of the Declaration of Helsinki, and all patients provided written informed consent. Patients were screened at the time of an acute Gouty Arthritis flare; eligible patients were subsequently randomized and received either canakinumab at one of five doses (10, $25,50,90$, or $150 \mathrm{mg}$ ) by subcutaneous (s.c.) injection and saline by intramuscular (i.m.) injection, or i.m. triamcinolone acetonide $(40 \mathrm{mg})$ and a s.c. placebo injection on day 1 . Randomization was carried out by means of an interactive voice response system. Patients were not informed of their assigned treatment during the study; wherever possible, treatment was administered by an unblinded pharmacist, nurse or physician who was not involved in any of the study assessments, allowing the investigator to be blinded to treatment. This was the case for 161 of the 200 patients (80.5\%) who thus received double-blind treatment.

Patients recorded pain intensity at pre-specified time points (using a Likert scale and visual analog scale (VAS)) and their use of rescue medication during the first seven days of the study. Patients who had difficulty tolerating their pain after the six-hour post-dose pain assessments could take rescue medication consisting of prednisone to a maximum dose of $30 \mathrm{mg}$ once daily for up to five days and acetaminophen $500 \mathrm{mg}$ (up to a maximum of $1 \mathrm{~g} /$ dose or $3 \mathrm{~g} /$ day) and/or codeine $30 \mathrm{mg}$ (up to a maximum of $30 \mathrm{mg} /$ dose or $180 \mathrm{mg} /$ day) as needed during the first seven days, but not within four hours before a pain assessment. Patients returned to the study center three days ( 72 hours after study drug administration) seven days, four weeks, and eight weeks post-dose for efficacy and safety assessments. Patients were not informed of their assigned treatment during the study; physicians were not blinded to treatment.

\section{Patients}

Key inclusion criteria were: patients aged 18 to 80 years with a history of at least one previous Gouty Arthritis 
flare and meeting the American College of Rheumatology 1977 preliminary criteria for the classification of acute arthritis of primary gout; presence of an acute Gouty Arthritis flare for no longer than five days; baseline pain intensity $\geq 50 \mathrm{~mm}$ on the 0 to $100 \mathrm{~mm}$ VAS; unresponsive or intolerant to, or contraindicated for NSAIDs and/or colchicine; body mass index (BMI) $\leq 40 \mathrm{~kg} / \mathrm{m}^{2}$. Unresponsiveness and intolerance to NSAIDs and/or colchicine and contraindication for NSAIDs and/or colchicine were based on physicians' assessment. Patients on urate-lowering therapy (ULT) were required to be on a stable dose and schedule, with no changes in therapy for four weeks before randomization, and were to be expected to remain on a stable regimen during study participation.

Exclusion criteria included: use of prohibited medications before screening (any ibuprofen in the 4 hours before screening (day 1 ) or $>400 \mathrm{mg}$ in the 8 hours before screening; any acetaminophen in the 4 hours before screening or $>1 \mathrm{~g}$ in the 24 hours before screening; any aspirin in the 4 hours before screening or $>600 \mathrm{mg}$ in the 24 hours before screening; any over-the-counter analgesic aspirin-based or acetaminophen-based combination medication tablets in the 4 hours before screening or $>2$ tablets in the 24 hours before screening; any diclofenac in the 8 hours before screening or $>50 \mathrm{mg}$ in the 24 hours before screening; any naproxen in the 12 hours before screening or $>500 \mathrm{mg}$ in the 24 hours before screening; cyclo-oxygenase- 2 inhibitors in the 48 hours before screening; other NSAIDs in the 24 hours before screening; systemic corticosteroids in the 24 hours before screening (a dose $<10 \mathrm{mg}$ of prednisolone or equivalent was permissible in the 24 hours before screening); intraarticular corticosteroids in the 4 weeks before screening; more than one dose of $0.6 \mathrm{mg}$ colchicine in the 24 hours before screening, if not on a stable dose regimen; anakinra in the 24 hours before screening; rilonacept in the week before screening; other investigational drugs or experimental biologic treatment, other than anakinra or rilonacept, in the 30 days (or 3 months for monoclonal antibodies) or five half-lives before screening, whichever was longer, or as instructed by local regulations; any tumor necrosis factor inhibitor in the 3 months before randomization; rheumatoid, infectious/septic or other inflammatory arthritis; severe renal function impairment; drug allergies; idiopathic thrombocytopenic purpura; contraindication to i.m. injections; donation or loss of $\geq 400$ $\mathrm{mL}$ of blood in the 8 weeks before dosing; live vaccination in the 3 months before the start of the study; known presence or suspicion of active or recurrent infection at enrolment; evidence of active pulmonary disease; requirement for administration of antibiotics against latent tuberculosis; risk factors for tuberculosis; any surgical or underlying hepatic, hematological, pulmonary, infectious or gastrointestinal condition that compromised the patient's immune system and/or placed them at unacceptable risk if they received immunomodulatory therapy; long QT syndrome or QTc $>450 \mathrm{~ms}$ for men and $>470$ $\mathrm{ms}$ for women at screening or baseline; significant medical problems, e.g. uncontrolled hypertension, uncontrolled diabetes, thyroid disease, history of malignancy of any organ system within the preceding 5 years; pregnant or nursing (lactating) women; women who were physiologically capable of becoming pregnant unless they were using an acceptable method of contraception; familial and social conditions rendering regular medical assessment impractical.

\section{Assessment and definition of response}

Study assessments made at baseline and each subsequent scheduled clinic visit (72 hours, 7 days, 4 weeks, and 8 weeks post-dose) included: patient assessment of pain intensity using a 5-point Likert scale (recording no (0), mild (1), moderate (2), severe (3), and extreme (4) pain) and a VAS (ranging from no pain $(0 \mathrm{~mm})$ to unbearable pain $(100 \mathrm{~mm})$ ); the physician's assessment of tenderness, swelling, and erythema in the target joint; and physician and patient global assessments of response to treatment. In addition, blood samples were collected for assessment of blood chemistry (including CRP and SAA levels) and hematology. Adverse events (AEs) were reported throughout the study and physicians assessed local tolerability at sites of s.c. and i.m. injections at each scheduled visit. Blood samples were assessed for anti-canakinumab antibodies at baseline and at eight weeks post-dose using a validated Biacore ${ }^{\circledR}$ binding assay (Biacore International AB, Uppsala, Sweden) [25].

Pain intensity scores (according to the Likert scale and VAS) were recorded by patients in their diaries at 6 , 12,24 , and 48 hours, and 4, 5, and 6 days post-dose, and during scheduled clinic visits at 72 hours, 7 days, 4 weeks, and 8 weeks post-dose. Physicians assessed inflammation in the target joint using the following tenderness and swelling scales: tenderness rated as none, 'no pain'; mild, 'pain'; moderate, 'pain and winces'; severe, 'pain; winces and withdraws'; and swelling rated as none, 'no swelling'; mild, 'palpable'; moderate, 'visible'; and severe, 'bulging beyond the joint margins'. Erythema was assessed as 'absent', 'present' or 'not assessable'. Physicians rated response to treatment as 'very good', 'good', 'fair', 'poor', or 'very poor'; and patients rated response to treatment as 'excellent', 'good', 'acceptable', 'slight' or 'poor'. Subsequent flares were identified from patient-reported signs and symptoms of Gouty Arthritis.

\section{HRQoL instruments}

HRQoL was assessed at baseline, and at seven days and eight weeks post-dose using the SF-36 (acute version 2) 
and the Health Assessment Questionnaire (HAQ). These were exploratory endpoints.

\section{6-item Short-Form Health Survey}

SF-36 measures the impact of disease on overall quality of life and consists of eight individual domains that can be grouped to derive a physical component summary (PCS) (composed of physical functioning, role-physical, bodily pain and general health) and a mental component summary (MCS) (composed of vitality, social functioning, role-emotional and mental health) [26]. Scores range from 0 to 100 , where 0 represents the worst possible health and 100 is perfect health [27]. This instrument has been validated for use in patients with Gouty Arthritis $[28,29]$. This study employed the acute (oneweek) recall version of SF-36 version 2 [30]. This more recently developed acute form of the 36-item questionnaire was designed for applications in which health status is measured weekly or biweekly. It was created by changing the recall period for six of the eight scales [Role-Physical (RP), Bodily Pain (BP), Vitality (VT), Social Functioning (SF), Role-Emotional (RE) and Mental Health (MH)] from "the past four weeks" to "the past week". The other two scales, Physical Functioning (PF) and General Health (GH), do not have a recall period; the items and instructions for these scales are identical across acute and standard forms. This acute version of SF-36 has been shown to be more sensitive to recent changes in health status. Results were plotted as spidergrams, as recommended by Strand et al. [31].

\section{Health Assessment Questionnaire}

The HAQ assesses a patient's physical ability, functional status, and quality of life through 20 questions concerning difficulty in performing eight common activities of daily living [32,33]. Patients choose from four response categories with scoring of 0 to 3 , ranging from 'without any difficulty' (0) to 'unable to do' (3).

\section{Statistical analysis}

In this paper we report the results of secondary efficacy endpoints with respect to reduction of signs and symptoms of inflammation during flares and exploratory endpoints regarding HRQoL. Results for the primary endpoint and for other secondary endpoints have been reported previously and are not included in this paper [24].

Differences in the reduction in pain (Likert scale) were analyzed using an analysis of covariance with treatment group, baseline Likert scale score, and baseline BMI as covariates, while differences in joint tenderness, swelling and erythema were assessed using proportional odds regression with treatment group, baseline physician assessment, and baseline BMI as covariates. Differences in physician and patient global assessments were assessed using proportional odds regression with treatment group and BMI at baseline as covariates. The percentage of patients with no/mild pain was analyzed using a logistical regression model with baseline Likert score and BMI as covariates. The percentage of patients with normalized CRP and SAA concentration values was analyzed using a logistical regression model with baseline CRP/SAA concentration value and BMI as covariates. Mean and standard deviation (SD) were determined for SF-36 PCS, MCS and subscale scores. Descriptive analyses are provided for HAQ scores. All covariates were defined a priori. No adjustment was made for multiplicity.

All efficacy end points were analyzed using the full analysis set (i.e. all randomized patients who received study drug had at least one post-baseline VAS assessment) and safety assessments were based on the safety analysis set (i.e. all randomized patients who received study drug and had at least one post-baseline safety assessment).

\section{Results}

Between November 2008 and May 2009, 200 patients from 89 centers in 11 countries (Argentina, Belgium, Canada, France, Germany, Poland, Russia, Switzerland, Turkey, the UK and the USA) were enrolled and 191 patients completed the study (Figure 1). The demographic and baseline disease characteristics of patients enrolled on this study were generally well balanced across treatment groups and have been described previously [24] (for details see Supplementary table S1 in Additional file 1). Most patients had had multiple acute Gouty Arthritis flares in the preceding 12 months (mean number of flares in each treatment group, 3.9 to 6.8). There was a baseline imbalance in pain intensity between groups, with mean scores being lowest in the canakinumab $150 \mathrm{mg}$ group; the imbalance was significant for VAS score $(P=0.005)$, but not for the Likert scale scores. Physician baseline assessments of joint tenderness, swelling, and erythema were generally well balanced among treatment groups.

\section{Pain reduction}

At baseline, 98\% of patients were suffering from moderate, severe, or extreme pain in the target joint (Likert assessment) (see Supplementary table S1 in Additional file 1). Reductions in the percentage of patients with moderate/severe/extreme pain were seen in all treatment groups from six hours post-dose onwards. The percentage of patients with no or mild pain was numerically greater in most canakinumab groups compared with triamcinolone acetonide from 24 to 72 hours postdose and the difference was statistically significant for the $150 \mathrm{mg}$ group at these time points $(P<0.05)$ (Figure 2). The reduction in pain intensity from baseline was also significantly greater for canakinumab $150 \mathrm{mg}$ 


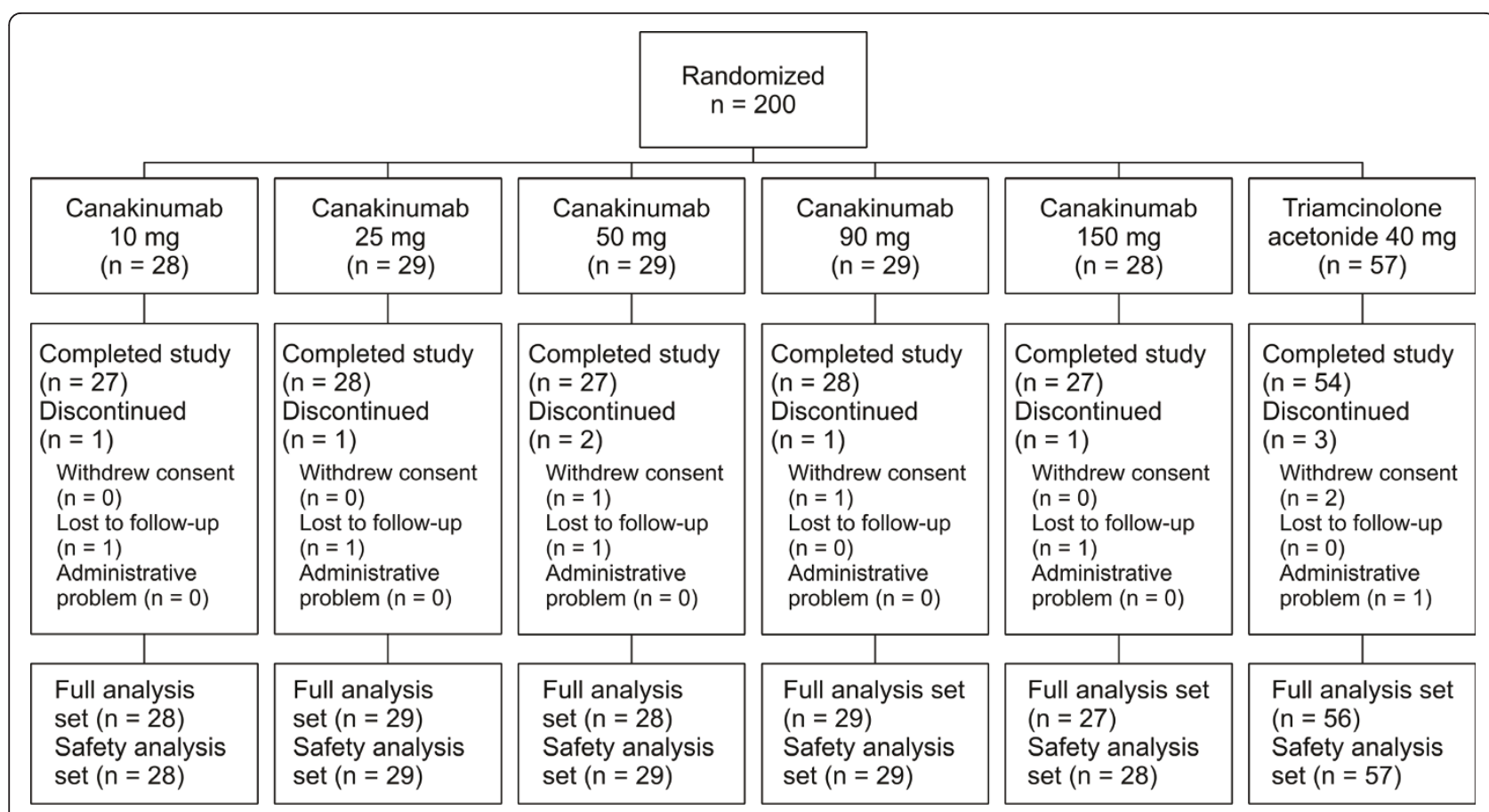

Figure 1 Study disposition. Number of patients who entered and completed the study and reasons for discontinuation.

compared with triamcinolone acetonide from 48 hours post-dose to 7 days post-dose (Figure 2).

\section{Reduction in signs of inflammation Physician and patient assessments}

At baseline, most patients had moderate or severe tenderness (85\% of patients), moderate or severe joint swelling ( $85 \%$ of patients), and/or erythema ( $83 \%$ of patients). All treatments reduced visible signs of inflammation in the target joint by 72 hours post-dose (the first assessment). At this time point, patients treated with canakinumab $150 \mathrm{mg}$ had a statistically significant lower score on the Likert scale for tenderness and for swelling compared with patients receiving triamcinolone acetonide and the difference between treatments remained statistically significant at seven days post-dose (Figures 3 and 4). Erythema was absent in $74.1 \%$ of patients receiving canakinumab $150 \mathrm{mg}$ and $69.6 \%$ of patients receiving triamcinolone acetonide at 72 hours post-dose and in $96.3 \%$ (canakinumab $150 \mathrm{mg}$ ) and $83.9 \%$ (triamcinolone acetonide) of patients at seven days post-dose. At 72 hours post-dose canakinumab 150 mg was also associated with statistically significant better responses to treatment according to patient global self-assessment and physician global assessment compared with patients treated with triamcinolone acetonide (Figure 4).

\section{Inflammatory markers}

At baseline, CRP levels and SAA levels were above the upper limit of the normal range in the majority of patients (CRP, 79.2\%; SAA, 64.0\%). At seven days postdose, CRP levels were normalized $(\leq 3.0 \mathrm{mg} / \mathrm{L})$ in 46.4 to $72.4 \%$ of patients in the canakinumab groups vs $41.1 \%$ in the triamcinolone acetonide group (Table 1). For the canakinumab $150 \mathrm{mg}$ group, the percentage of patients with normalized CRP levels was significantly greater than that in the triamcinolone acetonide group at seven days, four weeks, and eight weeks post-dose $(P<0.05)$. SAA levels were normalized $(\leq 6.7 \mathrm{mg} / \mathrm{L})$ in 63.0 to $75.9 \%$ of canakinumab-treated patients compared with $44.6 \%$ of patients receiving triamcinolone acetonide at seven days post-dose (Table 1 ). From this time point onwards the percentage of patients with normalized SAA levels was numerically greater for all canakinumab groups compared with triamcinolone acetonide, but the difference did not reach statistical significance for most doses or time points (Table 1).

\section{HRQoL measures}

36-item Short-Form Health Survey (acute version 2)

At baseline, mean SF-36 PCS and mean MCS were well below those of the US general population: PCS, 30.0 to 36.1 (US general population, mean \pm SD $50.0 \pm 10.0$ ) and MCS, 42.9 to 48.2 (US general population, mean \pm 


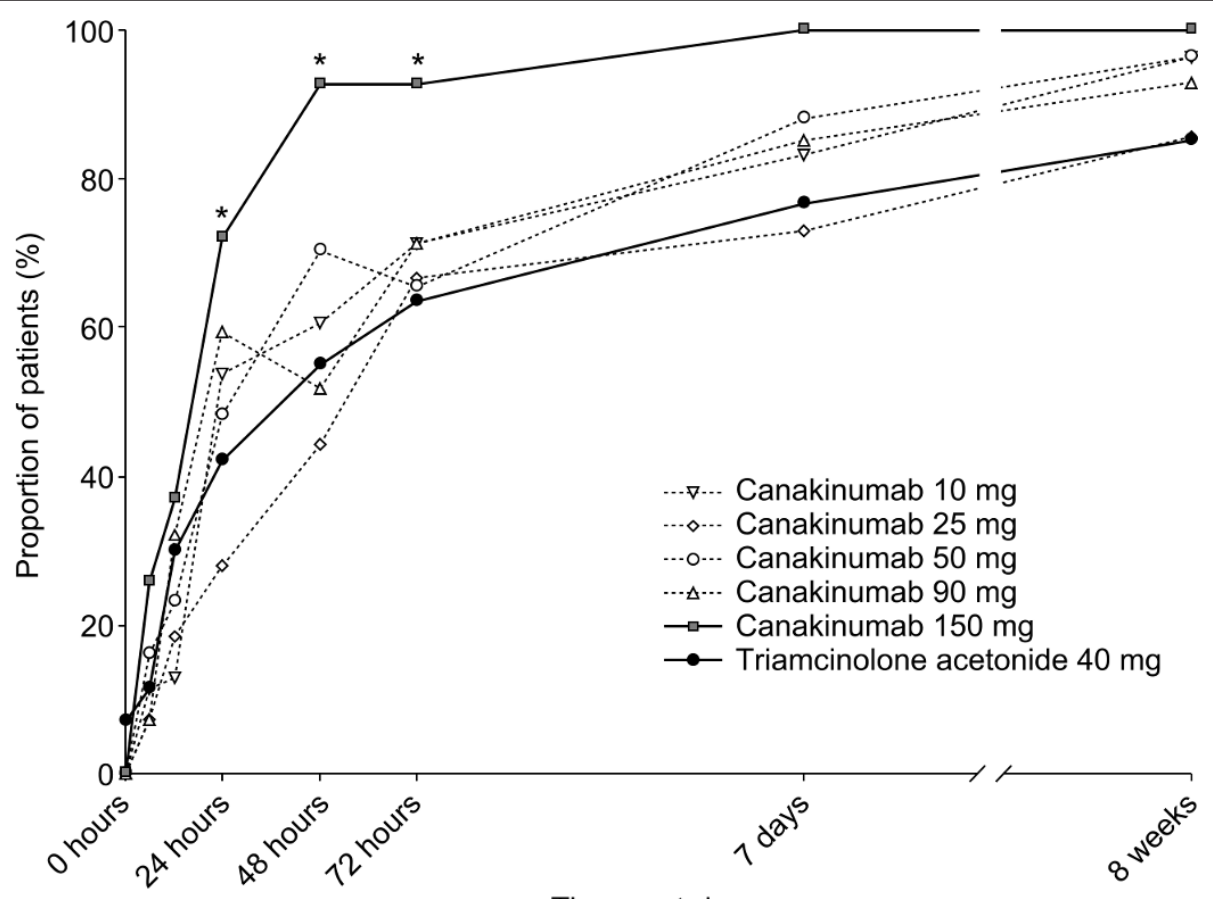

Time post-dose

Figure 2 Percentage of patients experiencing no or mild pain following administration of study medication. Pain assessments made using a 5-point Likert scale. ${ }^{*} P<0.05$ for canakinumab $150 \mathrm{mg}$ vs triamcinolone acetonide $40 \mathrm{mg}$. Cl, confidence interval; LS, least-squares.

SD $50.0 \pm 10.0$ ) [27]. Similarly mean scores for the individual SF-36 domains were much lower than those for the general US population: physical functioning, 31.1 to 41.5 (US general population, 84.2); role-physical, 31.3 to 53.0 (US general population, 80.9 ); bodily pain, 23.5 to 36.0 (US general population, 75.2); general health, 53.5 to 65.4 (US general population, 71.9); vitality, 41.3 to 53.9 (US general population, 60.9); social functioning, 47.7 to 62.5 (US general population, 83.3); role-emotional, 54.6 to 66.5 (US general population, 81.3); mental health, 58.1 to 67.9 (US general population, 74.7).

All aspects of physical health improved in all treatment groups over the first seven days post-dose, as reflected in increases in PCS, and were greatest for the canakinumab $150 \mathrm{mg}$ group. In this group, mean $( \pm$ SD) PCS increased by $12.0 \pm 10.0$ points from baseline to $48.3 \pm 8.6$ at seven days post-dose and exceeded that of the US general population by eight weeks post-dose, having a score of $52.8 \pm 6.7$. A more modest increase of $8.5 \pm 10.4$ points to $41.9 \pm 9.5$ at seven days post-dose was reported for the triamcinolone acetonide group and at eight weeks post-dose, the score $(47.1 \pm 11.2)$ remained below that of the US general population.

In the canakinumab $150 \mathrm{mg}$ group, the greatest improvements in physical function were seen in the physical functioning and bodily pain domains (Figure 5a). Mean physical functioning scores rapidly improved in the canakinumab $150 \mathrm{mg}$ group from 41.5 at baseline to 80.0 at seven days post-dose (a mean increase of 39.0 points), and exceeded the value for the US general population by eight weeks post-dose ( 86.1 vs 84.2 for the US general population). Similar improvements were seen in mean bodily pain scores in the canakinumab $150 \mathrm{mg}$ group from 36.0 at baseline to 72.2 at seven days postdose (a mean increase of 35.6 points) and 86.6 at eight weeks post-dose (vs 75.2 for the US general population). More modest and slower improvements were observed in the triamcinolone acetonide group and scores remained below those of the US general population at eight weeks post-dose (Figure 5b).

Improvements in mental well-being accompanied these changes in physical health. Mean MCS values increased in all treatment groups and exceeded that of the US general population at eight weeks post-dose in all canakinumab groups and approached that of the US general population for the triamcinolone acetonide group (mean \pm SD at eight weeks post-dose: canakinumab, $50.6 \pm 8.2$ to $53.3 \pm 7.4$; triamcinolone acetonide, $49.1 \pm 11.1)$. Improvements from baseline to eight weeks post-dose were greater in all canakinumab groups compared with triamcinolone acetonide (5.1 \pm 10.1 to $9.5 \pm 13.2$ vs $4.9 \pm 15.7)$. In the canakinumab $150 \mathrm{mg}$ group, improvements were seen in all individual domains, and the greatest improvement was seen 

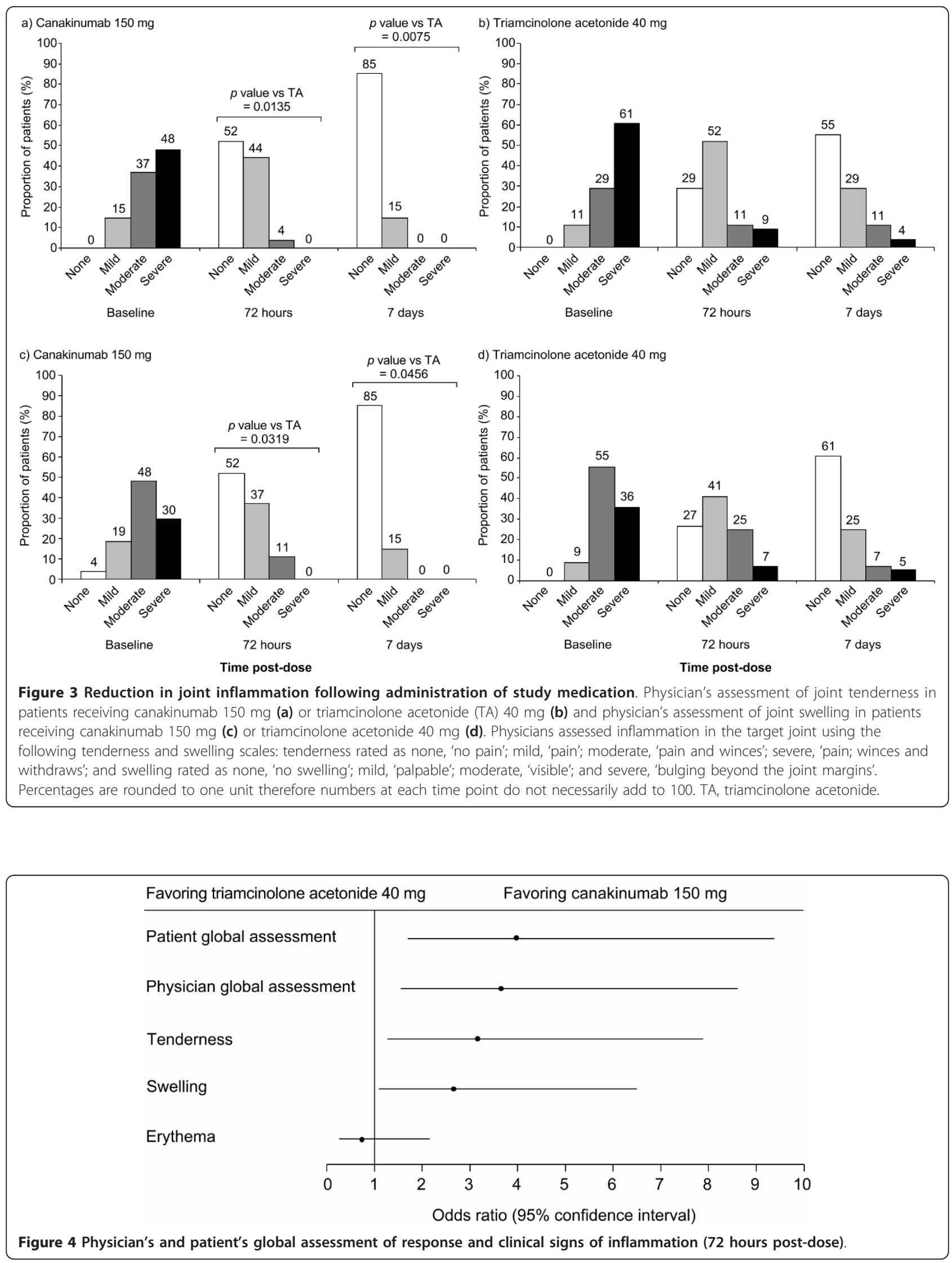
Table 1 Percentage of patients achieving normalization ${ }^{1}$ of C-reactive protein levels and serum amyloid A protein levels

\begin{tabular}{|c|c|c|c|c|c|c|}
\hline Variable & $\begin{array}{c}\text { Canakinumab } \\
10 \mathrm{mg} \\
N=28\end{array}$ & $\begin{array}{c}\text { Canakinumab } \\
25 \mathrm{mg} \\
N=29\end{array}$ & $\begin{array}{c}\text { Canakinumab } \\
50 \mathrm{mg} \\
N=28\end{array}$ & $\begin{array}{c}\text { Canakinumab } \\
90 \mathrm{mg} \\
N=29\end{array}$ & $\begin{array}{c}\text { Canakinumab } \\
\begin{array}{c}150 \mathrm{mg} \\
N=27\end{array}\end{array}$ & $\begin{array}{c}\text { Triamcinolone } \\
\text { acetonide } 40 \mathrm{mg} \\
\quad N=56\end{array}$ \\
\hline \multicolumn{7}{|l|}{ CRP } \\
\hline Baseline & $4(14.3)$ & $8(27.6)$ & $6(21.4)$ & $6(20.7)$ & $5(18.5)$ & $12(21.4)$ \\
\hline 3 days post-dose & $6(22.2)$ & $13(46.4)$ & $9(34.6)$ & $9(32.1)$ & $11(44.0)$ & $19(35.8)$ \\
\hline 7 days post-dose & $13(46.4)$ & $21(72.4)^{*}$ & $16(57.1)$ & $19(67.9)^{*}$ & $19(70.4)^{*}$ & $23(41.8)$ \\
\hline 4 weeks post-dose & $21(77.8)^{*}$ & $22(78.6)^{*}$ & $20(74.1)^{*}$ & $18(64.3)$ & $20(74.1)^{*}$ & $27(49.1)$ \\
\hline 8 weeks post-dose & $17(65.4)^{*}$ & $21(75.0)^{*}$ & $17(63.0)$ & $21(75.0)^{*}$ & $22(81.5)^{*}$ & $23(42.6)$ \\
\hline \multicolumn{7}{|l|}{$\overline{\mathrm{SAA}}$} \\
\hline Baseline & $8(28.6)$ & $9(32.1)$ & $9(32.1)$ & $8(27.6)$ & $9(33.3)$ & $23(44.2)$ \\
\hline 3 days post-dose & $15(57.7)$ & $14(50.0)$ & $16(57.1)$ & $13(46.4)$ & $13(48.1)$ & $26(48.1)$ \\
\hline 7 days post-dose & $19(67.9)$ & $22(75.9)^{*}$ & $19(67.9)$ & $21(75.0)^{*}$ & $17(65.4)$ & $25(47.2)$ \\
\hline 4 weeks post-dose & $24(88.9)^{*}$ & $24(82.8)$ & $19(70.4)$ & $20(71.4)$ & $18(69.2)$ & $34(63.0)$ \\
\hline 8 weeks post-dose & $18(69.2)$ & $21(80.8)$ & $18(69.2)$ & $19(70.4)$ & $20(74.1)$ & $30(57.7)$ \\
\hline
\end{tabular}

* $P<0.05$ vs triamcinolone acetonide $40 \mathrm{mg}$.

${ }^{1}$ Upper limit of normal: $3 \mathrm{mg} / \mathrm{L}$ for CRP, $6.7 \mathrm{mg} / \mathrm{L}$ for SAA.

CRP, C-reactive protein; SAA, serum amyloid $A$ protein

for social functioning (Figure 5a). An increase of 18.8 points from baseline to 81.7 was seen at seven days post-dose for the canakinumab $150 \mathrm{mg}$ group, and a further increase to 91.7 was seen at eight weeks post-dose, which exceeded that of the US general population (83.3). Improvements in social functioning were also seen in the triamcinolone acetonide group but the mean score remained below that of the US general population at eight weeks post-dose (78.1 vs 83.3) (Figure 5b).

\section{Health Assessment Questionnaire}

Mean HAQ scores at baseline were indicative of mild functional disability and were similar for all groups except the canakinumab $150 \mathrm{mg}$ group (mean for all groups except canakinumab $150 \mathrm{mg}, 1.03$ to 1.28 ; canakinumab $150 \mathrm{mg}, 0.74 ; P=0.063)$. Reductions in disability were seen in all canakinumab and triamcinolone acetonide groups, reflected in reductions in HAQ score from baseline of 0.46 to 0.67 at seven days post-dose and 0.52 to 0.85 at eight weeks post-dose.

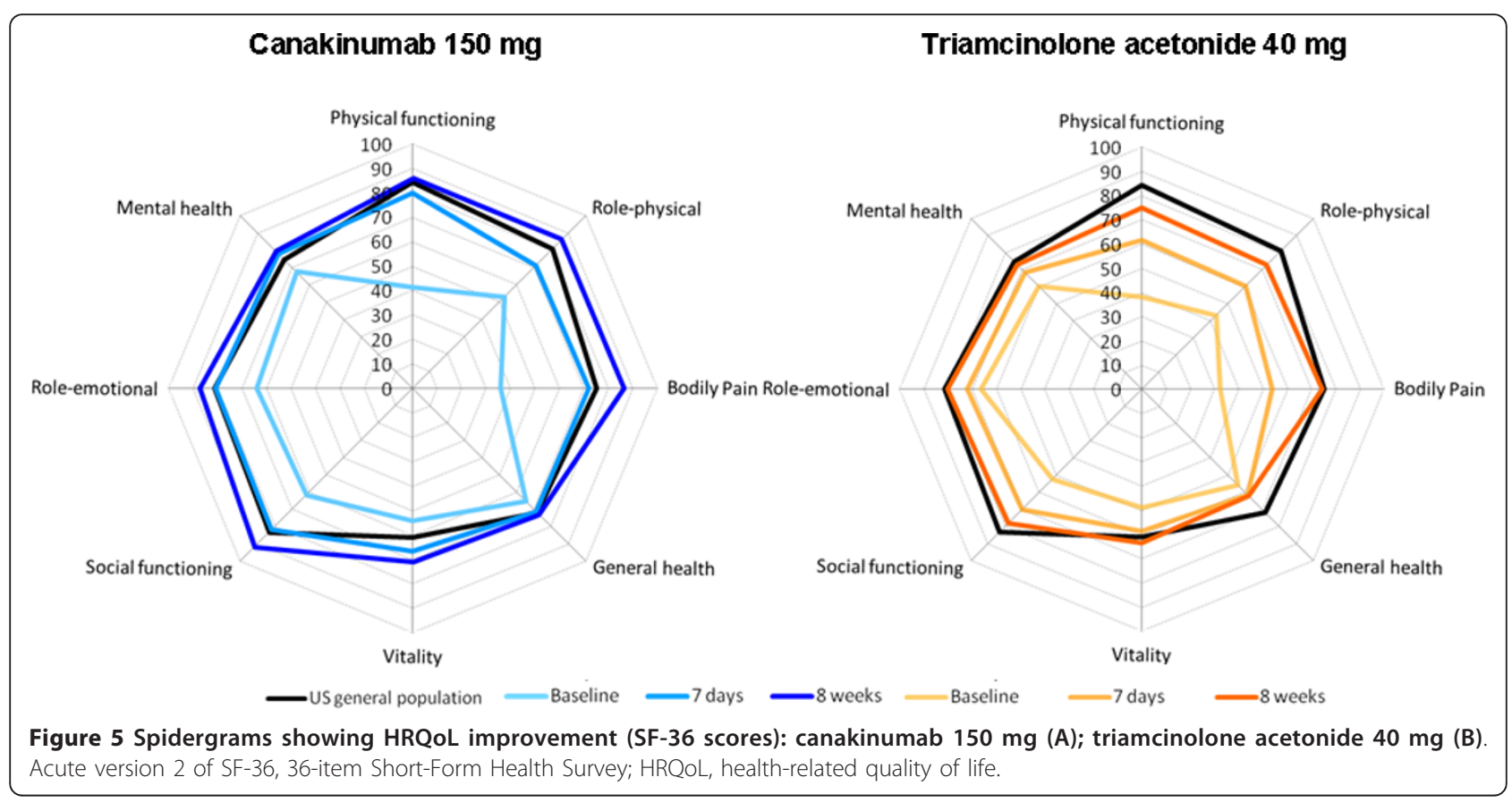




\section{Safety and tolerability}

As reported previously [24], all treatments were generally well tolerated. There were no deaths and no patients experienced serious AEs related to the study drugs or discontinued the study owing to AEs. The incidence of patients with AEs was similar for canakinumab (59 out of 143 patients, $41.3 \%$ ) and triamcinolone acetonide (24 out of 57 patients, $42.1 \%$ ), and all except two of the AEs were mild or moderate in severity. The incidence of infections was low; $7 \%$ of all canakinumab-treated patients and $7 \%$ of those in the triamcinolone acetonide group. Anti-canakinumab antibodies were detected in two patients in the canakinumab $150 \mathrm{mg}$ group. In one patient, antibodies were detected at baseline, but were absent at the end of the study. In the second patient, antibodies were detected at baseline and at the end of the study. No anti-canakinumab antibodies were detected in the other treatment groups.

\section{Discussion}

Gouty Arthritis causes severe pain and morbidity $[34,35]$. In our study, $98 \%$ of patients reported having moderate-to-extreme pain at baseline and $68 \%$ of patients reported having severe or extreme pain; as reported previously, mean VAS scores at baseline were 66 to $78 \mathrm{~mm}$ [24]. These data are in broad agreement with other studies reporting on the severity of pain during an acute flare and indicate clearly that the majority of patients experience severe pain [36-39]. For example, two other studies which have assessed pain in patients with acute Gouty Arthritis using a 5-point Likert scale have reported that 49 to $53 \%$ [38] and $89 \%$ of patients had severe or extreme pain at baseline [36], whereas two studies which have employed a 0 to $100 \mathrm{~mm}$ VAS reported baseline scores of 59 to $62 \mathrm{~mm}$ [37] and 74 to $78 \mathrm{~mm}$ during activity [39]. These scores suggest that pain associated with acute flares is at least as great or greater than that experienced by patients with osteoarthritis (OA) (mean VAS score, $68 \mathrm{~mm} \mathrm{[40])} \mathrm{or} \mathrm{rheuma-}$ toid arthritis (RA) (mean VAS scores of 64 to $67 \mathrm{~mm}$ [40] and $62 \mathrm{~mm}$ [41] have been reported). Rapid effective pain relief is, therefore, a priority for management of acute Gouty Arthritis.

In our study, canakinumab treatment produced rapid and sustained reductions in pain in patients with acute Gouty Arthritis who were unresponsive or intolerant to, or contraindicated for NSAIDs and/or colchicine. Reductions in pain according to Likert scale scores were seen with all canakinumab doses, with 24 to $67 \%$ of patients having no or mild pain by 24 hours post-dose (compared with $38 \%$ with triamcinolone acetonide), and reductions in pain in the canakinumab $150 \mathrm{mg}$ group were significantly greater than those reported for triamcinolone acetonide from 48 hours to 7 days post-dose.
These results paralleled those we have previously reported for our study using the 0 to $100 \mathrm{~mm}$ VAS [24]. Pain relief was rapid, with the LS mean reduction in pain at 48 hours post-dose being 2.0 (according to the Likert scale) and $58.0 \mathrm{~mm}$ (according to the VAS assessment) in the canakinumab $150 \mathrm{mg}$ group.

Comparison of results across different studies necessarily needs to be cautious given differences in study design and patient population. However, a meaningful comparison can be made with the results reported by Janssens et al. who assessed pain relief in a similar patient population using a 0 to $100 \mathrm{~mm}$ VAS scale at 12 hour intervals for up to 90 hours following the first dose of treatment [37]. In this double-blind study, patients were randomized to receive prednisolone $35 \mathrm{mg}$ once daily or naproxen $500 \mathrm{mg}$ twice daily. Pain scores at baseline were approximately $15 \mathrm{~mm}$ less than in our study, suggesting that pain was slightly more severe in our study. Reductions in pain score from baseline were comparable for prednisolone and naproxen at all time points and were less than those reported in our study for canakinumab doses of $50 \mathrm{mg}$ or greater or for triamcinolone acetonide at the corresponding times. For example, the mean change from baseline was $10 \mathrm{~mm}$ for prednisolone and $8 \mathrm{~mm}$ for naproxen at zero to six hours [37], and $20 \mathrm{~mm}$ for canakinumab $150 \mathrm{mg}$ and $12 \mathrm{~mm}$ for triamcinolone acetonide at six hours in our study. Similarly, at 66 to 78 hours the mean change from baseline was $42 \mathrm{~mm}$ for both prednisone and naproxen, comparable with the $43 \mathrm{~mm}$ change from baseline observed with triamcinolone acetonide $40 \mathrm{mg}$ at 72 hours in our study; a change from baseline of $63 \mathrm{~mm}$ was observed at 72 hours for canakinumab $150 \mathrm{mg}$. The robust pain reductions described here are in contrast to those reported for another inhibitor of IL-1 $\beta$ signaling in development, which has apparently failed to demonstrate significant improvements in pain (relative to a standard regimen of indomethacin) in patients with acute Gouty Arthritis [42].

Triamcinolone acetonide $40 \mathrm{mg}$ was chosen as the comparator in this study based on the experience of the investigators and the fact that in two countries in which the study was performed, the $40 \mathrm{mg}$ im dose is labelled as the initial dose or usual dose and higher doses were not considered to be acceptable to investigators or the health authorities. Furthermore, according to a recent survey, $72 \%$ of prescriptions for triamcinolone acetonide in France, Germany and the UK in 2008 to 2009 were for the $40 \mathrm{mg}$ dosage (IMS Disease Analyser: Prescriptions of triamcinolone acetonide in France, and Germany for August 2009 to August 2010 and in the UK for December 2008 to December 2009, personal communication). However, there are no comparative trials to indicate that the $60 \mathrm{mg}$ dose is more effective than 
the $40 \mathrm{mg}$ dose. The results of our study indicate that triamcinolone acetonide $40 \mathrm{mg}$ is an effective treatment for acute Gouty Arthritis having at least comparable efficacy to that reported for prednisolone and naproxen by Janssens et al. [37]. Thus triamcinolone acetonide $40 \mathrm{mg}$ was an appropriate comparator to use in this study.

The results reported here for canakinumab are in agreement with accumulating evidence suggesting that IL- $1 \beta$, in addition to mediating inflammation, can stimulate pain directly by activating nociceptors (pain receptors) [43] and indirectly by signaling through complex cascades that upregulate and/or activate other pain stimulating molecules [44]. Moreover, IL-1 $\beta$ release in response to injury can contribute to persistent pain by stimulating neural hyperexcitability and increasing sensitivity to pain (hyperalgesia) [44]. Therefore, inhibition of IL- $1 \beta$ with canakinumab may lead directly to a reduction in pain, as well as indirectly through inhibition of inflammation.

The Special Interest Group for gout outcomes at the Outcome Measures in Rheumatology Clinical Trials (OMERACT) recognized the importance of HRQoL measurement in gout and included it as a core domain for clinical trials for chronic Gouty Arthritis [45]. Undoubtedly, the pain associated with acute flares has a severe impact on quality of life, as was evident in our study and has been reported previously [17-21,46]. We herein report the use of the HRQoL instruments SF-36 acute version and HAQ in acute Gouty Arthritis patients. In our study, mean baseline scores for all SF-36 physical domains and for the PCS were considerably lower than those for the US general population (physical functioning, 31 to 42; role-physical, 31 to 53; bodily pain, 24 to 36; PCS, 30 to 36). These scores suggest considerably reduced physical function and are comparable with those expected for men in the US general population aged $>75$ years, while most of our study population was of working age with an approximate median age of 50 years. Baseline scores for $\mathrm{HAQ}$ were indicative of moderate disability, and were in agreement with the reduced physical function evident from SF-36 scores.

The influence of gouty arthritis on patient HRQoL is becoming increasingly recognized but still there are limited data available for HRQoL during an acute flare. Our results are in agreement with a number of recent studies using the SF-36 scale to assess the HRQoL of Gouty Arthritis patients with differing disease severities $[18,19,21,28]$. Interestingly, the scores reported in our study are similar to those reported in two studies using patients who were intolerant of or refractory to ULT (who would be expected to have frequent flares) $[18,21]$. For example, Becker et al. reported a mean SF-36 physical functioning score of 46.8 , a mean role-physical score of 35.0 and PCS value of 34.2 for a population with a mean age of 59 years and experiencing 0.6 flares per month [18], while Strand et al. reported reductions of 30 to 32 points for physical function, role-physical and bodily pain SF-36 domains compared with age- and gendermatched controls [21]. Furthermore, in our study, all aspects of mental health were reduced to lower than US norms and were in agreement with scores reported by Strand et al. These authors suggested that the HRQoL for their patient population was comparable with that of patients suffering from long-standing RA or active systemic lupus erythematosus, and was much lower than that for patients with OA or cardiac angina [21]. This outcome provides initial insights into the impact of acute Gouty Arthritis flares on HRQoL in an acute setting, which has not been studied so far. Long-term follow up of HRQoL in Gouty Arthritis patients, using other more specialized HRQoL questionnaires, such as the EQ-5D, may yield further insights into the long-term outcomes of the different treatments in the future.

In all groups, treatment was associated with dramatic improvements in HRQoL, particularly relating to physical function, by seven days post-dose. The greatest improvements were seen in the physical functioning and bodily pain domains, and were particularly marked in the canakinumab $150 \mathrm{mg}$ group. By seven days postdose, mean scores almost reached or were equivalent to those for the US general population for the canakinumab $150 \mathrm{mg}$ group on all domains and reached or exceeded scores for the general population on most domains by eight weeks post-dose. In contrast, in the triamcinolone acetonide group, mean scores remained 10 to 20 points below those of the general population at seven days post-dose and approached those of the general population by eight weeks post-dose. This is the first study to report the impact of anti-inflammatory therapy on quality of life in patients with acute Gouty Arthritis and demonstrates the significant value of potent anti-inflammatory therapy.

We also report that canakinumab achieved rapid reductions in inflammation. Physician-assessed visible signs of inflammation in the joint were reduced by 72 hours post-dose following treatment with canakinumab, and greater reductions in joint tenderness and swelling were observed throughout the study with canakinumab $150 \mathrm{mg}$ compared with triamcinolone acetonide. In addition, treatment with canakinumab $150 \mathrm{mg}$ was associated with a statistically significant better response to treatment according to patient global selfassessment $(P=0.002)$ and physician global assessment $(P=0.003)$ compared with patients treated with triamcinolone acetonide. Previous studies of NSAIDs and corticosteroids have reported the effects of treatment on visible signs of inflammation [36,38,47-50]. However, these studies have used different scales to measure 
effects and hence results are not comparable with those we report here.

Rapid reductions in visible signs of inflammation were accompanied by significant reductions in levels of the main acute phase inflammatory proteins, CRP and SAA. By seven days post-dose, CRP and SAA levels were normalized in 70\% (CRP) and 63\% (SAA) of patients receiving canakinumab $150 \mathrm{mg}$. This supports evidence indicating that IL- $1 \beta$ contributes to the regulation of the production of CRP and SAA [51]. By inhibiting the activity of IL- $1 \beta$ and its generation from pro-IL- $1 \beta$, canakinumab decreases the production of CRP and SAA. This may be clinically important, not only for its impact on acute Gouty Arthritis and the risk of recurrent flares, but also because of the possible role of elevated CRP and SAA levels and IL-1 $\beta$ in the development of cardiovascular disease [52-54]. As Gouty Arthritis may be an independent risk factor for coronary artery disease [55] and for cardiovascular mortality [56], reducing the levels of these inflammatory proteins may be of additional benefit in patients with Gouty Arthritis.

Our study has a number of limitations. First, the study involved patients who were unresponsive or intolerant to or contraindicated for NSAIDs and/or colchicine, based on their physician's assessment. Physicians made this assessment based on an interview with the patient and from the patient's medical history. We chose to ask physicians to make these judgments so that the study reflects routine clinical practice and would provide an insight into the characteristics of patients for whom physicians consider standard anti-inflammatory therapy to be inappropriate. Second, in this early phase II study the effect on HRQoL was included as an explorative objective. This was limited to assessments made in an acute setting using the acute version 2 of SF-36 and the HAQ. Further studies including HRQoL assessments targeting longerterm outcomes and in specific patient populations will be required in the future. Third, the study was open to patients who were within five days of the onset of their flare. Thus by 72 hours post-dose, it could be expected that the flare would have started to resolve independently of treatment in some patients. However, the proportion of patients for whom this could apply was very small as the majority of patients (81\%) entered the study within three days of onset of flare. Furthermore, the proportion of patients entering the study within four or five days of onset of flare was similar across treatment groups. Thus the differences between canakinumab $150 \mathrm{mg}$ and triamcinolone acetonide observed in this study reflect differences in the efficacy of the two treatments.

\section{Conclusions}

Acute Gouty Arthritis flares are often associated with excruciating pain that can be associated with disability and reduced physical functioning. The results of this study indicate that anti-inflammatory therapy with canakinumab $150 \mathrm{mg}$ produces rapid pain relief, relieves signs and symptoms of inflammation and results in rapid clinically significant improvements in quality of life, especially relating to physical function (as assessed using the SF-36 acute version 2). These results are particularly important for patients with acute Gouty Arthritis who are unable to achieve adequate responses to standard therapies, or to tolerate standard therapies. The canakinumab $150 \mathrm{mg}$ dose is being investigated further in ongoing phase III clinical trials.

\section{Additional material}

Additional file 1: Supplementary Table S1: Demographic and baseline characteristics. Table giving demographic and baseline characteristics of the study population by treatment group.

\section{Abbreviations}

AE: adverse event; BMI: body mass index; Cl: confidence interval; CRP: Creactive protein; HAQ: Health Assessment Questionnaire; HRQoL: healthrelated quality of life; IL-1 $\beta$ : interleukin-1 $\beta$; LS: least-squares; MCS: mental component summary; MSU: monosodium urate; NSAID: non-steroidal antiinflammatory drug; OA: osteoarthritis; OMERACT: Outcome Measures in Rheumatology Clinical Trials; PCS: physical component summary; RA: rheumatoid arthritis; SAA: serum amyloid A protein; SF-36: 36-item ShortForm Health Survey; ULN: upper limit of the normal range; ULT: uratelowering therapy; VAS: visual analog scale.

\section{Acknowledgements}

The authors thank the patients and investigators (the Canakinumab in Gout Study Group) who took part in this study. The authors take full responsibility for the content of the paper. They thank Alberto Gimona (Senior Global Program Medical Director, Integrated Hospital Care Franchise, Novartis Pharma AG) and Alberto Ferreira (Associate Director, Health Economics and Outcomes Research, Novartis Pharma AG) for critical review of the manuscript and helpful suggestions, and Kirstin Stricker (Clinical Communication Leader, a member of the clinical development team, Novartis Pharma AG) for her contribution to the interpretation of the data, critical review of the paper and for coordinating author discussions and writing of the manuscript. They also thank Rowena Hughes and Gemma Carter (Oxford PharmaGenesis ${ }^{\mathrm{TM}}$ Ltd) for medical writing support, editorial assistance, and collation and incorporation of comments from all authors. Such editorial help was supported by Novartis Pharma AG, Basel, Switzerland.

The following investigators were members of the Canakinumab in Gout Study Group: Argentina: Bernardo Pons Estel; Belgium: Marc De

Meulemeester; Canada: Dennis O'Keefe, Proton Rahman, John Li; France: Frederic Liote; Germany: Ingo Senftleber, René Martz, Oana Förster, Veit Lorenz, Ruediger Moericke, Uwe Gerbaulet, Hischam Bouzo; Poland: Maria Misterska-Skora; Russia: Evgeny Nasonov, Vadim Mazurov, Marianna Petrova, Andrey Pikhlak, Olga Ershova, Olga Ryabitseva, Anatoly Kuzin, Ludmila Suplotova; Switzerland: Claude Merlin, Burkhard Moeller, Alexander So, Alan Tyndall; Turkey: Yasar Karaaslan, Ayhan Dinç, Nurullah Akkoç, Taskin Sentürk, Süleyman Ozbek, Ahmet Onat, Ahmet Gül, A Eftal Yücel; UK: Bhavesh Bodalia, Adrian Darrah; USA: Kenneth Saag, Vishala Chindalore, Joel Silverfield, David Sikes, Wayne Gilbert, Billy Chacko, Michael Cox, William Knibbe, Howard Knapp, Chester Fisher, Elliott Schwartz, Mercedes Samson, Marina Fernandez, Charles White, Mohammad Ali, Paul Dura, Robert Trapp, Alan Kivitz, Atul Singhal, Andrew Baldassare, Lance Rudolph, Arnaldo Torres, Brock McConnehey, Daniel Ripley, Madura Rangaraj, Clancy Cone, Ara Dikranian, Naomi Schlesinger, Talha Shamim. 


\section{Author details}

'Division of Rheumatology, Department of Medicine, Robert Wood Johnson Medical School, 125 Patterson Street, New Brunswick, NJ 089010, USA. 2Pratique Médicale, Cabinet de Là-Haut, Rue de Marchienne 113, 6534 Gozée, Belgium. ${ }^{3}$ Moscow State University of Medicine and Dentistry, Clinical-Diagnostic Center MSMSU, Dolgorukovskaya Street 4, Moscow 127006, Russia. ${ }^{4}$ Baskent University, Faculty of Medicine, Baglica Kampusu, Eskisehir Yolu 20.km, Baglica 06530, Ankara, Turkey. ${ }^{5}$ Immunology \& Infectious Disease Therapeutic Area, Novartis Pharma AG, Lichtstrasse 35, CH4056, Basel, Switzerland. 'Service de Rhumatologie, Centre Hospitalier Universitaire Vaudois, University of Lausanne, Av. Pierre-Decker 5, CH-1005, Lausanne, Switzerland.

\section{Authors' contributions}

The study was designed by AS, NS, PS, DR, VM and UA, and data were gathered and analyzed by Novartis. All authors vouch for the accuracy of the data and the analysis, and contributed to the interpretation of the data and were involved in the decision to publish. All authors were involved in discussing the content of the manuscript and deciding which data and interpretations were to be included. All authors approved the submitted paper. The contribution of all those who do not meet the criteria for authorship are acknowledged in the paper.

\section{Competing interests}

Dr Schlesinger reports having received lecture fees from Takeda and grant support from the UMDNJ foundation and Novartis Pharmaceuticals as well as fees for serving on advisory boards: Novartis, Takeda, Savient, URL Pharma and EnzymeRx. Dr Pikhlak reports having received consulting fees from Novartis. Drs Sallstig, Richard, and Arulmani and Ms Murphy are employees of Novartis and report having equity interests in Novartis. Dr So reports having received consulting fees from Novartis, Wyeth, and Roche, having equity interests in Pfizer and having received lecture fees from Bristol Myers Squibb, and grant support from Fonds National Suisse. Drs De Meulemeester and Yücel declare that they have no competing interests.

Received: 24 September 2010 Revised: 11 February 2011 Accepted: 25 March 2011 Published: 25 March 2011

\section{References}

1. Schlesinger N: Diagnosis of gout: clinical, laboratory, and radiologic findings. Am J Manag Care 2005, 11:S443-450, quiz S465-448.

2. Schlesinger N: Diagnosis of gout. Minerva Med 2007, 98:759-767.

3. So A: Developments in the scientific and clinical understanding of gout. Arthritis Res Ther 2008, 10:221.

4. Martinon F, Petrilli V, Mayor A, Tardivel A, Tschopp J: Gout-associated uric acid crystals activate the NALP3 inflammasome. Nature 2006, 440:237-241.

5. Kim JH, Jin HM, Kim K, Song I, Youn BU, Matsuo K, Kim N: The mechanism of osteoclast differentiation induced by IL-1. J Immunol 2009, 183:1862-1870.

6. Tunyogi-Csapo M, Kis-Toth K, Radacs M, Farkas B, Jacobs JJ, Finnegan A, Mikecz K, Glant TT: Cytokine-controlled RANKL and osteoprotegerin expression by human and mouse synovial fibroblasts: fibroblastmediated pathologic bone resorption. Arthritis Rheum 2008, 58:2397-2408.

7. Schlesinger $N$, Thiele RG: The pathogenesis of bone erosions in gouty arthritis. Ann Rheum Dis 2010, 69:1907-1912.

8. Schlesinger N: Management of acute and chronic gouty arthritis: present state-of-the-art. Drugs 2004, 64:2399-2416.

9. Zhang W, Doherty M, Bardin T, Pascual E, Barskova V, Conaghan P, Gerster J, Jacobs J, Leeb B, Liote F, McCarthy G, Netter P, Nuki G, PerezRuiz F, Pignone A, Pimentao J, Punzi L, Roddy E, Uhlig T, ZimmermannGorska I: EULAR evidence based recommendations for gout. Part II: Management. Report of a task force of the EULAR Standing Committee for International Clinical Studies Including Therapeutics (ESCISIT). Ann Rheum Dis 2006, 65:1312-1324.

10. Schlesinger $\mathrm{N}$ : Overview of the management of acute gout and the role of adrenocorticotropic hormone. Drugs 2008, 68:407-415.

11. Petersel $\mathrm{D}$, Schlesinger $\mathrm{N}$ : Treatment of acute gout in hospitalized patients. J Rheumatol 2007, 34:1566-1568.

12. Schlesinger N, Schumacher R, Catton M, Maxwell L: Colchicine for acute gout. Cochrane Database Syst Rev 2006, CD006190.
13. Pascual E, Sivera F: Therapeutic advances in gout. Curr Opin Rheumatol 2007, 19:122-127.

14. Riedel AA, Nelson M, Wallace K, Joseph-Ridge N, Cleary M, Fam AG: Prevalence of comorbid conditions and prescription medication use among patients with gout and hyperuricemia in a managed care setting. J Clin Rheumatol 2004, 10:308-314.

15. Mandell BF: Clinical manifestations of hyperuricemia and gout. Cleve Clin J Med 2008, 75:S5-8.

16. Dalbeth N, Clark B, Gregory K, Gamble G, Sheehan T, Doyle A, McQueen FM: Mechanisms of bone erosion in gout; a quantitative analysis using plain radiography and computed tomography. Ann Rheum Dis 2009, 68:1290-1295.

17. Geletka RC, Hershfield MS, Scarlett EL, Sundy JS: Severe gout is associated with impaired quality of life and functional status. Arthritis Rheum 2004, 50:S340-S341.

18. Becker MA, Schumacher HR, Benjamin KL, Gorevic P, Greenwald M, Fessel J, Edwards L, Kawata AK, Frank L, Waltrip R, Maroli A, Huang B, Sundy JS: Quality of life and disability in patients with treatment-failure gout. $J$ Rheumatol 2009, 36:1041-1048.

19. Lee SJ, Hirsch JD, Terkeltaub R, Khanna D, Singh JA, Sarkin A, Kavanaugh A: Perceptions of disease and health-related quality of life among patients with gout. Rheumatology (Oxford) 2009, 48:582-586.

20. Singh JA: Quality of life and quality of care for patients with gout. Curr Rheumatol Rep 2009, 11:154-160.

21. Strand V, Edwards L, Singh JA: Health-related quality-of-life (HRQOL) of patients with treatment failure gout (TFG) is poor, and comparable to that in other severe chronic conditions. Ann Rheum Dis 2009, 68(Suppl 3):168,

22. Wu EQ, Yu AP, Guérin A, Latremouille-Viau D, Tsaneva M, Forsythe A: The costs of treatment failure gout: a claims-based analysis. 2009, Poster presented at 73rd Annual meeting of the American College Of Rheumatology, Philadelphia, PA, 17-21 October 2009, Abstract 1112.

23. Brook RA, Kleinman NL, Patel PA, Melkonian AK, Brizee TJ, Smeeding JE, Joseph-Ridge N: The economic burden of gout on an employed population. Curr Med Res Opin 2006, 22:1381-1389.

24. So A, De Meulemeester M, Pikhlak A, Yucel A, Richard D, Murphy V, Arulmani U, Sallstig P, Schlesinger N: Canakinumab for treatment of acute flares in refractory gouty arthritis. Arthritis Rheum 2010, 62:3064-3076.

25. Lachmann HJ, Kone-Paut I, Kuemmerle-Deschner JB, Leslie KS, Hachulla E, Quartier P, Gitton X, Widmer A, Patel N, Hawkins PN: Use of canakinumab in the cryopyrin-associated periodic syndrome. N Engl J Med 2009, 360:2416-2425.

26. Ware JE Jr, Sherbourne CD: The MOS 36-item short-form health survey (SF36). I. Conceptual framework and item selection. Med Care 1992, 30:473-483.

27. Ware JE, Kosinski M, Keller SD: SF36○ Physical and Mental Summary Scales: A User's Manual Boston: The Health Institute, New England Medical Center; 1994.

28. Singh JA, Strand V: Gout is associated with more comorbidities, poorer health-related quality of life and higher healthcare utilisation in US veterans. Ann Rheum Dis 2008, 67:1310-1316.

29. Khanna D, Ahmed M, Yontz D, Ginsburg SS, Park GS, Leonard A, Tsevat J: The disutility of chronic gout. Qual Life Res 2008, 17:815-822.

30. SF- $36^{\circledR}$ Health Survey Update. [http://www.sf-36.org/tools/sf36.shtml].

31. Strand V, Crawford B, Singh J, Choy E, Smolen JS, Khanna D: Use of "spydergrams" to present and interpret SF-36 health-related quality of life data across rheumatic diseases. Ann Rheum Dis 2009, 68:1800-1804.

32. Pincus T, Summey JA, Soraci SA Jr, Wallston KA, Hummon NP: Assessment of patient satisfaction in activities of daily living using a modified Stanford Health Assessment Questionnaire. Arthritis Rheum 1983, 26:1346-1353.

33. Fries JF, Spitz P, Kraines RG, Holman HR: Measurement of patient outcome in arthritis. Arthritis Rheum 1980, 23:137-145.

34. Martinon F, Glimcher LH: Gout: new insights into an old disease. J Clin Invest 2006, 116:2073-2075.

35. Choi HK, Curhan G: Gout: epidemiology and lifestyle choices. Curr Opin Rheumatol 2005, 17:341-345.

36. Cheng TT, Lai HM, Chiu CK, Chem YC: A single-blind, randomized, controlled trial to assess the efficacy and tolerability of rofecoxib, diclofenac sodium, and meloxicam in patients with acute gouty arthritis. Clin Ther 2004, 26:399-406.

37. Janssens HJ, Janssen M, van de Lisdonk EH, van Riel PL, van Weel C: Use of oral prednisolone or naproxen for the treatment of gout arthritis: a double-blind, randomised equivalence trial. Lancet 2008, 371:1854-1860. 
38. Willburger RE, Mysler E, Derbot J, Jung T, Thurston H, Kreiss A, Litschig S, Krammer G, Tate GA: Lumiracoxib 400 mg once daily is comparable to indomethacin $50 \mathrm{mg}$ three times daily for the treatment of acute flares of gout. Rheumatology (Oxford) 2007, 46:1126-1132.

39. Man CY, Cheung IT, Cameron PA, Rainer TH: Comparison of oral prednisolone/paracetamol and oral indomethacin/paracetamol combination therapy in the treatment of acute goutlike arthritis: a double-blind, randomized, controlled trial. Ann Emerg Med 2007, 49:670-677.

40. Schnitzer TJ, Gitton X, Jayawardene S, Sloan VS: Lumiracoxib in the treatment of osteoarthritis, rheumatoid arthritis and acute postoperative dental pain: results of three dose-response studies. Curr Med Res Opin 2005, 21:151-161.

41. Pasero G, Marcolongo R, Serni U, Parnham MJ, Ferrer F: A multi-centre, double-blind comparative study of the efficacy and safety of aceclofenac and diclofenac in the treatment of rheumatoid arthritis. Curr Med Res Opin 1995, 13:305-315.

42. Neogi T: IL-1 antagonism in acute gout: Is targeting a single cytokine the answer? Arthritis Rheum 2010, 62:2845-2849.

43. Opree A, Kress M: Involvement of the proinflammatory cytokines tumor necrosis factor-alpha, IL-1 beta, and IL- 6 but not IL-8 in the development of heat hyperalgesia: effects on heat-evoked calcitonin gene-related peptide release from rat skin. J Neurosci 2000, 20:6289-6293.

44. Ren $K$, Torres $R$ : Role of interleukin-1 beta during pain and inflammation. Brain Res Rev 2009, 60:57-64.

45. Schumacher HR, Taylor W, Edwards L, Grainger R, Schlesinger N, Dalbeth N, Sivera F, Singh J, Evans R, Waltrip RW, Diaz-Torne C, MacDonald P, McQueen F, Perez-Ruiz F: Outcome domains for studies of acute and chronic gout. I Rheumatol 2009, 36:2342-2345.

46. Roddy E, Zhang W, Doherty M: Is gout associated with reduced quality of life? A case-control study. Rheumatology (Oxford) 2007, 46:1441-1444.

47. Rubin BR, Burton R, Navarra S, Antigua J, Londono J, Pryhuber KG, Lund M, Chen E, Najarian DK, Petruschke RA, Ozturk ZE, Geba GP: Efficacy and safety profile of treatment with etoricoxib $120 \mathrm{mg}$ once daily compared with indomethacin $50 \mathrm{mg}$ three times daily in acute gout: a randomized controlled trial. Arthritis Rheum 2004, 50:598-606.

48. Schumacher HR Jr, Boice JA, Daikh DI, Mukhopadhyay S, Malmstrom K, $\mathrm{Ng}$ J, Tate GA, Molina J: Randomised double blind trial of etoricoxib and indometacin in treatment of acute gouty arthritis. BMJ 2002, 324:1488-1492.

49. Alloway JA, Moriarty MJ, Hoogland YT, Nashel DJ: Comparison of triamcinolone acetonide with indomethacin in the treatment of acute gouty arthritis. J Rheumatol 1993, 20:111-113.

50. Siegel LB, Alloway JA, Nashel DJ: Comparison of adrenocorticotropic hormone and triamcinolone acetonide in the treatment of acute gouty arthritis. J Rheumatol 1994, 21:1325-1327.

51. Berger P, McConnell JP, Nunn M, Kornman KS, Sorrell J, Stephenson K, Duff GW: C-reactive protein levels are influenced by common IL-1 gene variations. Cytokine 2002, 17:171-174.

52. Berliner JA, Navab M, Fogelman AM, Frank JS, Demer LL, Edwards PA, Watson AD, Lusis AJ: Atherosclerosis: basic mechanisms. Oxidation, inflammation, and genetics. Circulation 1995, 91:2488-2496.

53. Clearfield MB: C-reactive protein: a new risk assessment tool for cardiovascular disease. J Am Osteopath Assoc 2005, 105:409-416.

54. Duewell P, Kono H, Rayner K, Sirois CM, Vladimer G, Bauernfeind FG, Abela GS, Franchi L, Nunez G, Schnurr M, Espevik T, Lien E, Fitzgerald KA, Rock KL, Moore KJ, Wright SD, Hornung V, Latz E: NLRP3 inflammasomes are required for atherogenesis and activated by cholesterol crystals. Nature 2010, 464:1357-1361.

55. Krishnan E, Svendsen K, Neaton JD, Grandits G, Kuller LH: Long-term cardiovascular mortality among middle-aged men with gout. Arch Intern Med 2008, 168:1104-1110.

56. Choi HK, Curhan G: Independent impact of gout on mortality and risk for coronary heart disease. Circulation 2007, 116:894-900.

doi:10.1186/ar3297

Cite this article as: Schlesinger et al:: Canakinumab relieves symptoms of acute flares and improves health-related quality of life in patients with difficult-to-treat Gouty Arthritis by suppressing inflammation: results of a randomized, dose-ranging study. Arthritis Research \& Therapy 2011 13:R53.

\section{Submit your next manuscript to BioMed Central and take full advantage of:}

- Convenient online submission

- Thorough peer review

- No space constraints or color figure charges

- Immediate publication on acceptance

- Inclusion in PubMed, CAS, Scopus and Google Scholar

- Research which is freely available for redistribution 